\title{
Die Vertragsreformen von Lissabon in den Bereichen Inneres und Justiz: verstärkte Handlungsfähigkeit, Kontrolle und Differenzierung
}

\author{
Jörg Monar*
}

\section{Entwicklungsdynamik und Probleme der EU-Innen- und Justizpolitik}

Die seit dem Vertrag von Amsterdam unter dem grundlegenden Vertragsziel der Schaffung eines „Raumes der Freiheit, der Sicherheit und des Rechts“ (RFSR) zusammengefassten Bereiche Inneres und Justiz gehören seit 1999 zu den am stärksten expandierenden Politikbereichen der Union: Allein im Jahre 2007 hat der Rat „Inneres und Justiz“ nicht weniger als 164 Texte angenommen, ${ }^{1}$ es vergeht keine Sitzung des Europäischen Rates mehr ohne die Aufnahme langer Abschnitte zu innen- und justizpolitischen Themen in die Schlussfolgerungen der Präsidentschaft ${ }^{2}$ und auch die Haushaltslinien für diesen Bereich verzeichnen überdurchschnittliche Zuwachsraten. ${ }^{3}$ An der politischen Bedeutung dieser Handlungsfelder kann auch nicht gezweifelt werden, handelt es sich doch um nicht weniger als drei grundlegende öffentliche Güter, die dem EU-Bürger im Rahmen des RFSR geboten werden sollen: ,Freiheit' (primär im Sinne von Freizügigkeit), ,Sicherheit' (primär im Sinne von Schutz gegen schwerwiegende grenzüberschreitende Kriminalität) und ,Recht' (primär im Sinne der Sicherstellung der grenzüberschreitenden Wirkung richterlicher Entscheidungen und des grenzüberschreitenden Zugangs zum Recht). Nach Eurobarometer-Umfragen sind die Zustimmungsraten der Bürger zu einer verstärkten Rolle der Union, zumindest in einigen Feldern der Innen- und Justizpolitik - wie etwa der Bekämpfung von Terrorismus und organisierter Kriminalität ${ }^{4}$ - außerordentlich hoch, was den wiederholten Hinweisen des Europäischen Rates auf die ,hohe Priorität“, die die europäischen Bürger dem RFSR anscheinend zumessen, ${ }^{5}$ eine gewisse Berechtigung gibt.

Dennoch steht fast zehn Jahre nach seiner Einführung durch den Amsterdamer Vertrag mit dem Integrationsziel und großen politischen Projekt des RFSR nicht alles zum Besten: Die Proliferation von Aktionsplänen, Strukturen und Instrumenten kann nicht darüber hinwegtäuschen, dass viele gesetzte Ziele nicht oder nur teilweise erreicht worden sind und die Umsetzung beschlossener Maßnahmen oft sehr zu wünschen übrig lässt. Es genügt, einen Blick in die jährlichen Berichte der Kommission zur Umsetzung des 2005 bis 2010 laufenden Haager Programms zur Weiterentwicklung des RFSR ${ }^{6}$ oder die halbjährlichen Evaluierungsberichte des

Prof. Dr. Dr. Jörg Monar, College of Europe, Brügge; Sussex European Institute, University of Sussex, Brighton.

1 Information der Generaldirektion $\mathrm{H}$ des Generalsekretariats des Rates der EU.

2 Siehe beispielsweise die Schlussfolgerungen der jüngsten Tagung des Europäischen Rates: Rat der Europäischen Union: Tagung des Europäischen Rates vom 19./20. Juni 2008 in Brüssel. Schlussfolgerungen des Vorsitzes, Dok. 11018/1/08 REV 1 vom 17. Juli 2008, Paragraphen 6-24.

3 Im Haushaltsjahr 2008 16,7\% gegenüber 2007, und 2007 12,8\% gegenüber 2006 (Quelle: Europäische Kommission: Gesamthaushaltsplan der Europäischen Union für das Haushaltsjahr 2008 (und 2007), Brüssel 2008 und 2007).

$481 \%$ im EU-Durchschnitt gemäß Europäische Kommission: Special Eurobarometer 290: The role of the European Union in Justice, Freedom and Security policy areas, Juni 2008, S. 6-7.

5 Siehe beispielsweise Rat der Europäischen Union: Tagung des Europäischen Rates vom 19./20. Juni 2008, Paragraph 6.

6 Der jüngste: Europäische Kommission: Mitteilung der Kommission an den Rat und das Europäische Parlament. Bericht über die Umsetzung des Haager Programms im Jahr 2007, KOM(2008) 373. 


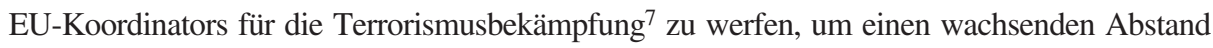
zwischen Anspruch und Wirklichkeit bei der Umsetzung der hehren Zielsetzungen des RFSR festzustellen. Nach Kalkulationen der Kommission vom Juli 2008 wurden 2007 nur 38 Prozent der Zielsetzungen des Haager Programms gemäß den ursprünglichen Zeitvorgaben erreicht, mit extrem niedrigen Fortschrittsquoten in Schlüsselbereichen wie der Asyl- und Visapolitik und polizeilichen Zusammenarbeit sowie erheblichen Umsetzungsdefiziten auch auf der nationalen Ebene. ${ }^{8}$ Damit nicht genug, haben die in den Neunzigerjahren noch eher vereinzelten kritischen Töne hinsichtlich der möglichen Gefährdung von Menschen- und Bürgerrechten vor dem Hintergrund der sich vervielfältigenden EU-Datenaustauschsysteme, Restriktionen im Bereich der Asyl- und Einwanderungspolitik und strafrechtlichen Maßnahmen mittlerweile geradezu orchestrale Dimensionen angenommen und der Forderung nach besserer parlamentarischer und richterlicher Kontrolle einen beträchtlichen Auftrieb verliehen, dem sowohl das Europäische Parlament als auch viele einzelstaatlichen Parlamente die notwendige politische Kraft verleihen.

Der Vertrag von Lissabon bot eine hervorragende Möglichkeit, auf diese offenkundigen Entwicklungsprobleme des RFSR durch Reformen der vertraglichen Grundlagen zu reagieren. Zumindest quantitativ scheint diese Herausforderung auch angenommen worden zu sein, übertrifft die Zahl der den RFSR betreffenden - größtenteils aus dem Verfassungsvertrag übernommenen - Einzelreformen doch die jedes anderen einzelnen Politikbereichs der Union. Die eigentliche Frage aber muss natürlich lauten, inwieweit der neue Vertrag tatsächlich qualitative Veränderungen bringt, die zu einer Überwindung der angedeuteten Probleme und einer effektiven Weiterentwicklung der EU-Innen- und Justizpolitik dienen können. Diese Frage ist auch im Falle eines definitiven Scheiterns des Vertragswerkes nicht müßig, da die bei ihrer Beantwortung aufgewiesenen Probleme und möglichen Entwicklungspotenziale in der einen oder anderen Form notwendigerweise auf der Agenda des RFSR bleiben werden.

Gemäß den oben angedeuteten gegenwärtigen primären Entwicklungsproblemen des RFSR wird dieser Artikel sich zunächst mit den Reformen, mit Bedeutung für die Handlungsfähigkeit der Union und deren parlamentarische und richterliche Kontrolle beschäftigen, um dann auf die erhöhte ,Differenzierung ' einzugehen, die zu den wesentlichen Kosten dieser Reformen gehört. Hieran schließen sich einige Schlussfolgerungen zu den weiteren Entwicklungsperspektiven des RFSR mit und ohne den Vertrag von Lissabon an.

\section{Verstärkte Handlungsfähigkeit}

War die Innen- und Justizpolitik durch den Vertrag von Amsterdam bereits unter dem neuen Leitziel des RFSR in die Reihe der grundlegenden Vertragsziele des Artikels 2 EUVertrag (EUV) aufgerückt, so setzt der Vertrag von Lissabon deren formalen ,Aufstieg ' insoweit fort, als der RFSR im neugefassten Artikel 3(2) EUV-Lissabon ${ }^{9}$ nunmehr unter den grundlegenden Vertragszielen von der vierten auf die zweite Position aufrückt. ${ }^{10}$ Zudem

7 Der jüngste: Rat der Europäischen Union: Implementation of the EU Counter-Terrorism Strategy - Priorities for further action, Dok. 9417/08 vom 19. Mai 2008 sowie Rat der Europäischen Union: Implementation of the Strategy and Action Plan to Combat Terrorism, Dok. 9416/1/08 REV 1 und Dok. 9416/08 ADD 1 REV 1 vom 26. Mai 2008.

8 Siehe Europäische Kommission: Bericht über die Umsetzung des Haager Programms im Jahr 2007, 2008, insbesondere S. 2-3 und 14-16.

9 Zur Unterscheidung vom geltenden EUV werden Vertragsartikel gemäß der konsolidierten Fassung des Vertrags über die Europäische Union von Lissabon vom 9. Mai 2008 hier und im Folgenden als ,EUV-Lissabon“ zitiert. Die entsprechende konsolidierte Fassung des Vertrags über die Arbeitsweise der Europäischen Union wird als AEUV zitiert.

10 Unmittelbar nach dem Ziel, ,,den Frieden, ihre Werte und das Wohlergehen ihrer Völker zu fördern“ (Artikel 3 EUV-Lissabon). Bislang gingen dem RFSR die Ziele der Förderungen des wirtschaftlichen und sozialen Fortschritts, die Behauptung ihrer Identität auf internationaler Ebene und die Stärkung des Schutzes der Rechte und Interessen der Angehörigen ihrer Mitgliedstaaten durch Einführung einer Unionsbürgerschaft voraus. 
„,bietet“ die Union den RFSR nunmehr den „Bürgerinnen und Bürgern“ anstatt diesen nur zu „erhalten“. Damit wird erstmals ein ausdrücklicher Bezug zwischen der Union als dem Gewährleister des RFSR und den Bürgern als den Begünstigten hergestellt, was gleichfalls als eine zumindest politisch-symbolische Aufwertung betrachtet werden kann. Dieser Aufwertung entsprechen eine Reihe von Reformen, die direkt oder indirekt zur Steigerung der Handlungsfähigkeit der Union in diesem Bereich beitragen können:

\section{Die (unvollständige) Aufhebung der Säulenstruktur}

Im Rahmen der neuen Vertragsarchitektur werden die Materien der bisherigen Titel IV EG-Vertrag (Asyl, Einwanderung, justizielle Zusammenarbeit in Zivilsachen, Grenzkontrollen) und VI EUV (polizeiliche und justizielle Zusammenarbeit in Strafsachen - die bisherige ,dritte Säule') in einem neuen Titel V des dritten Teils des AEUV zusammengezogen. Dies kommt einer faktischen ,Vergemeinschaftung' der bisherigen ,dritten Säule‘ gleich, da für deren Materien als Ergebnis dieser Zusammenführung die Rechtsgrundlagen und -instrumente sowie Entscheidungsverfahren des bisherigen EG-Vertrages (EGV) zur Anwendung kommen. Hierdurch wird die bisherige ,Säulenteilung ' mit der artifiziellen rechtlichen Trennung der betreffenden Handlungsbereiche formell aufgehoben. Damit wird unter anderem die Notwendigkeit zur Annahme ,paralleler' Rechtsakte im Rahmen beider ,Säulen“ im Falle , säulenübergreifender` Materien (wie etwa bei der Bekämpfung der Geldwäsche) aufgehoben, das Potenzial für Konflikte hinsichtlich der Rechtsgrundlage reduziert ${ }^{11}$ und die Aushandlung von Abkommen mit Drittstaaten zu , säulenübergreifenden' Materien erleichtert. Mit der Zusammenführung der gegenwärtigen Bereiche des Titels IV EGV und des Titels VI EUV im selben Vertrag wird auch ein Beitrag zur Rechtssicherheit und Transparenz im Rahmen des RFSR geleistet, da fortan die gleichen Rechtsinstrumente für alle Handlungsbereiche zur Verfügung stehen und die Union auch international als einheitlich und einzig handelnde Rechtspersönlichkeit auftreten kann. Da die bisherige ,säulenbedingte Komplexität des RFSR zu Verzögerungen im Entscheidungsprozess, einigen interinstitutionellen Konflikten über Rechtsgrundlagen und gelegentlichen Irritationen bei Drittstaaten geführt hat, kann die im Vertrag vorgesehene formelle Aufhebung der bisherigen Trennung zwischen ,erster" und ,dritter Säule ' durchaus einen positiven Beitrag zur Handlungsfähigkeit der Union in der Innen- und Justizpolitik leisten.

Der ursprünglich durch den Verfassungsvertrag vorgesehene einheitliche vertragliche Rahmen wird allerdings nicht erreicht: Das grundlegende Vertragsziel des RFSR (Artikel 3(2) EUV-Lissabon) und die Charta der Grundrechte bleiben von den ,operativen“ Bestimmungen des AEUV durch den separaten vertraglichen Rahmen des EU-Vertrages getrennt. Während dies kaum Auswirkungen auf die Handlungsfähigkeit der Union haben dürfte, ist die Trennung der ,operativen' Bestimmungen des RFSR in Titel V des ersten Teils des AEUV von denen des Titels V EUV-Lissabon über das auswärtige Handeln der Union und die Gemeinsame Außen- und Sicherheitspolitik (GASP) vom Gesichtspunkt der Handlungsfähigkeit zu bedauern: Zur Erreichung der Ziele des RFSR - vor allem hinsichtlich der Reduzierung illegaler Einwanderung und der Bekämpfung von Terrorismus und organisierter Kriminalität - bedient sich die Union in stark zunehmendem Maße auch auswärtiger Hand-

11 Bei Maßnahmen im strafrechtlichen Bereich haben die Mitgliedstaaten beispielsweise bislang mehr oder weniger systematisch Rechtsgrundlagen der, dritten Säule‘ vorgezogen, was unter anderem zu einem Konflikt mit der Kommission über strafrechtliche Maßnahmen im Bereich des Umweltschutzes führte, der vom EuGH 2005 zugunsten der Kommission und einer gemeinschaftsrechtlichen Rechtsgrundlage entschieden wurde (Europäischer Gerichtshof: Rs. C-176/03 (Kommission/Rat), Slg. 2005, I-7879). 
lungsformen unter Einschluss spezifischer GASP-Verfahren und -instrumente (politische Dialoge mit Drittstaaten, koordinierte Positionen in internationale Organisationen, usw.), die Terrorismusbekämpfung ist spätestens seit der „Europäischen Sicherheitsstrategie“ vom Dezember 2003 zum , säulenübergreifenden' Sicherheitsziel erhoben worden und der Kampf gegen organisierte Kriminalität und Terrorismus sowie der Aufbau von wirksamen Polizeiund Justizstrukturen gehören zu den wichtigsten Zielen vieler gemeinsamer Operationen im Rahmen der Europäischen Sicherheits- und Verteidigungspolitik (ESVP). ${ }^{12}$ Angesichts dieser zunehmenden materiellen Verschränkung der Außendimension des RFSR mit GASPund ESVP-Zielsetzungen kann die fortbestehende vertragliche Trennung von RFSR und GASP nur weiterhin Komplexität in der Entscheidungsfindung, gelegentliche interinstitutionelle Konflikte und Konfusion bei Drittstaaten bedeuten.

\section{Die (teilweise) Reform der Entscheidungsverfahren}

In ihren Fortschrittsberichten zur Umsetzung des Haager Programms - und auch in ihrem Vorschlag vom Juni 2006, die , dritte Säule“ durch Nutzung der ,Passerelle ' des Artikels 42 EUV zu , vergemeinschaften " - hat die Kommission (neben den unterschiedlichen Rechtsgrundlagen und -instrumenten) wiederholt die für die Bereiche des Titels VI EUV geltende Einstimmigkeitserfordernis sowie auch das fehlende ausschließliche Initiativrecht als wesentliche Ursachen für die erheblichen Verzögerungen der Umsetzung des Programms in den betreffenden Bereichen identifiziert. ${ }^{13}$ Zwar hat die Kommission in ihrer konzentrierten Kritik der Entscheidungsverfahren der ,dritten Säule' die auch in den bereits, vergemeinschafteten " und größtenteils bereits Entscheidungen mit qualifizierter Mehrheit unterworfenen Bereichen der ,ersten Säule' durchaus verbreiteten Entscheidungsdefizite - wohl aus offenkundigem Eigeninteresse - etwas unterbelichtet, aber es kann kaum ein Zweifel daran bestehen, dass die Möglichkeit von Mehrheitsentscheidungen den Einigungsdruck und die Kompromissbereitschaft unter den einzelstaatlichen Delegationen im Rat erhöht.

Der Vertrag von Lissabon folgt der Logik der Kommission und anderer Befürworter einer ,Vergemeinschaftung ' der Entscheidungsverfahren im Bereich des RFSR indem er das gegenwärtige ,Mitentscheidungsverfahren ‘ mit ausschließlichem Initiativrecht der Kommission, qualifizierter Mehrheitsentscheidung im Rat und Mitentscheidung des Parlaments ${ }^{14}$ unter dem neuen Namen des „ordentlichen Gesetzgebungsverfahrens“ (Artikel 294 AEUV) - zum Standard-Entscheidungsverfahren im Bereich des RFSR erhebt. Damit werden Mehrheitsentscheidungen erstmals auch in den sensiblen Bereichen der polizeilichen und der justiziellen Zusammenarbeit in Strafsachen möglich - was als beträchtliche Neuerung zu werten ist und die Entscheidungsfähigkeit der Union entsprechend erhöhen könnte. Es gibt jedoch eine Reihe von nicht unwesentlichen Ausnahmen:

Wie gegenwärtig im Bereich des Titels VI EUV, wird die Kommission auch unter den Entscheidungsregeln von Lissabon über kein ausschließliches Initiativrecht in den Bereichen der polizeilichen und der justiziellen Zusammenarbeit in Strafsachen verfügen. Artikel 76 AEUV sieht vor, dass Gesetzgebungsvorschläge auch kollektiv von einem Viertel der Mitgliedstaaten eingebracht werden können. Dies erscheint als ein vertretbarer Kompro-

12 Als Beispiele seien die EU-Polizeimission in Bosnien-Herzegowina (EUPM), die EU-Rechtsstaatsmission im Kosovo (EULEX KOSOVO) und die EU-Polizeimission in Afghanistan (EUPOL AFGHANISTAN) genannt.

13 Siehe Europäische Kommission: Mitteilung der Kommission an den Rat und das Europäische Parlament. Umsetzung des Haager Programms: Weitere Schritte, $\operatorname{KOM}(2006)$ 331, S. 12; Mitteilung der Kommission an den Rat und das Europäische Parlament. Bericht über die Umsetzung des Haager Programms im Jahr 2006, $\operatorname{KOM}(2007)$ 373, S. 19; und Bericht über die Umsetzung des Haager Programms im Jahr 2007, 2008, S. 16-17.

14 Auf die verstärkte Beteiligung des Parlaments wird im Abschnitt „Verstärkte Kontrolle“ eingegangen. 
miss, wird hierdurch doch einerseits verhindert, dass Mitgliedstaaten einzelne rein von nationalen Interessen geprägte Vorschläge einbringen können, andererseits aber die Möglichkeit von kollektiven Initiativen mit genügender ,kritischer Masse ' hinsichtlich der Unterstützung im Rat eröffnet - die sich in der Vergangenheit, etwa beim Beschluss des Rates vom 28. Februar 2002 über die Errichtung von Eurojust, ${ }^{15}$ als der Entscheidungsfindung durchaus förderlich erwiesen hatten.

Schwerer unter den Ausnahmen wiegt jedoch, dass die Einstimmigkeitserfordernis im Rat für die folgenden Materien erhalten bleibt: für die Ausübung der Freizügigkeit relevante Bestimmungen betreffend Pässe, Personalausweise, Aufenthaltstitel oder diesen gleichgestellte Dokumente (Artikel 77(3) AEUV), Maßnahmen zum Familienrecht mit grenzüberschreitendem Bezug (Artikel 81(3) AEUV), Mindestvorschriften für „,sonstige“ (d.h. nicht bereits ausdrücklich vorgesehene) spezifische Aspekte des Strafverfahrensrechts (Artikel 82(2)(d) AEUV), die Bestimmung ,,anderer“ (d.h. nicht bereits ausdrücklich erwähnter) Bereiche schwerwiegender Kriminalität, für die der Rat Mindestvorschriften zur Festlegung von Straftaten und Strafen erlassen kann (Artikel 83(1) AEUV), die Einsetzung einer Europäischen Staatsanwaltschaft und die Ausweitung von deren Zuständigkeiten (Artikel 86(1) und (3) AEUV), Maßnahmen zur operativen Zusammenarbeit zwischen den Polizeibehörden der Mitgliedstaaten (Artikel 87(3) AEUV) und die Festlegung der Bedingungen und Grenzen innerhalb derer Polizeibehörden der Mitgliedstaaten im Hoheitsgebiet eines anderen Mitgliedstaats in Verbindung und in Absprache mit dessen Behörden tätig werden dürfen (Artikel 89 AEUV). Es bedarf keiner weiteren Erläuterung, dass es sich hier in der Tat um besonders sensible Materien hinsichtlich der einzelstaatlichen Souveränität und Territorialität handelt. Dennoch bleiben damit bedeutende nationale Vetomöglichkeiten in den Bereichen der bisherigen, dritten Säule ' erhalten - ein weiteres Relikt der formal aufgehobenen Säulenteilung.

Bleibt durch die Beibehaltung von ,Inseln der Einstimmigkeit' in den Materien des RFSR bereits eine Dimension bestehender Komplexität im Bereich der Entscheidungsverfahren erhalten, so wird diese Dimension im Vertrag von Lissabon durch zwei neue Verfahrensvarianten erheblich vergrößert:

Die erste Verfahrensvariante betrifft nur den strafrechtlichen Bereich. Bei Richtlinien zur Festlegung von Mindestvorschriften im Bereich des Strafverfahrensrechts (Artikel 82(3) AEUV) und bei Richtlinien zu Mindestvorschriften zur Festlegung von Straftaten und Strafen in Bereichen besonders schwerer Kriminalität (Artikel 83(3) AEUV), für die die qualifizierte Mehrheit zur Anwendung kommt, kann ein Mitgliedstaat, der der Auffassung ist, dass der betreffende Richtlinienentwurf ,grundlegende Aspekte seiner Strafrechtsordnung“ berührt, beantragen, dass der Europäische Rat befasst wird. In diesem Fall wird das ordentliche Gesetzgebungsverfahren ausgesetzt. ${ }^{16}$ Gelingt es dem Europäischen Rat nicht, binnen vier Monaten ein Einvernehmen herzustellen, so kann eine Gruppe von mindestens neun Mitgliedstaaten dem Europäischen Parlament, dem Rat und der Kommission mitteilen, dass sie die betreffende Richtlinie auf dem Wege einer ,verstärkten Zusammenarbeit“ annehmen möchte, woraufhin eine entsprechende Ermächtigung als gegeben gilt. Vielleicht in keiner anderen Neuerung des Vertrages wird so sehr deutlich, wie weit sich die Union mittlerweile von dem Gedanken einheitlicher prozeduraler und institutioneller Prinzipien entfernt hat. Tatsächlich bedeutet diese Variante, dass ein einzelner Mitgliedstaat im Falle der Berührung

15 Beschluss des Rates vom 28. Februar 2002 über die Errichtung von Eurojust zur Verstärkung der Bekämpfung der schweren Kriminalität, in: Amtsblatt der EG, Nr. L 63 vom 6. März 2002.

16 In den Verhandlungen hat sich hierfür der Begriff, Notbremse‘ etabliert. 
einzelstaatlicher Sonderinteressen ein normales der Mehrheitsentscheidung unterworfenes Gesetzgebungsverfahren aussetzen lassen kann - eine Möglichkeit, die einer Renaissance des sogenannten ,Luxemburger Kompromisses “ von 1966 im RFSR gleichkommt. Damit nicht genug, wird hier dem Europäischen Rat - dem nach der Logik der Verträge und der institutionellen Aufgabenteilung keine Funktion im ,normalen' Gesetzgebungsverfahren zukommt - eine ebensolche zugewiesen, hängt doch das Schicksal einer solchermaßen blockierten Richtlinie von den Verhandlungen im Europäischen Rat ab. Kommt es zu keiner Einigung, so wird gar die ursprünglich intendierte Aktionseinheit der Union durch den Übergang zur ,,verstärkten Zusammenarbeit“ aufgehoben. ${ }^{17}$ Es kann wohl davon ausgegangen werden, dass diese Verfahrensvariante aufgrund der enormen politischen Kosten für den sie auslösenden Mitgliedstaat nur in Extremfällen zur Anwendung gelangen würde, aber dennoch scheint die Frage gerechtfertigt, ob der Übergang zu Mehrheitsentscheidungen in den beiden genannten Strafrechtsmaterien ein solches Durchbrechen klarer prozeduraler und institutioneller Prinzipien rechtfertigen kann. Die Frage, ob derartige komplexe Verfahrensweisen dem ,Bürger auf der Straße‘ als dem angeblichen Begünstigten des RFSR jemals verständlich gemacht werden können, muss man gar nicht erst stellen.

Die zweite Verfahrensvariante besteht in der Möglichkeit einer Gruppe von mindestens neun Mitgliedstaaten, auf vereinfachtem Wege eine ,verstärkte Zusammenarbeit“ anzusteuern, falls im Rat keine Einstimmigkeit hinsichtlich der Einsetzung einer Europäischen Staatsanwaltschaft (Artikel 86(1) AEUV) oder der Maßnahmen zur operativen Zusammenarbeit zwischen den Polizeibehörden der Mitgliedstaaten (Artikel 87(3) AEUV) erreicht werden kann. ${ }^{18}$ In beiden Fällen kann die jeweilige 9+-Gruppe beantragen, dass der Europäische Rat mit dem entsprechenden Entwurf befasst wird. Gelingt es dem Europäischen Rat nicht, binnen vier Monaten ein Einvernehmen herzustellen, so kann die Gruppe dem Europäischen Parlament, dem Rat und der Kommission einfach mitteilen, dass sie die betreffende Maßnahme auf dem Wege einer ,,verstärkten Zusammenarbeit“ annehmen möchte, woraufhin eine entsprechende Ermächtigung als gegeben gilt. Auch hier wird wieder eine Möglichkeit zur Aushebelung eines normalen Gesetzgebungsverfahrens eröffnet und dem Europäischen Rat wieder ein wesentlicher Einfluss auf den Ausgang dieses Verfahrens eingeräumt. Diese zweite Variante ist im Wesentlichen eine Spiegelbildversion der ersten, mit dem einzigen Unterschied, dass in der zweiten eine Einstimmigkeitserfordernis umgangen werden kann und in der ersten eine Mehrheitserfordernis. Ganz offenkundig kennen sowohl die weniger als auch die mehr, integrationswilligen' ,Väter der Verträge ' mittlerweile kaum noch Grenzen hinsichtlich der Flexibilisierung von Prinzipien und Verfahrensweisen. Der einzig tröstliche Aspekt hier ist die Tatsache, dass ein solches Regelwerk den Bürgern nicht mehr unter dem Begriff ,Verfassung ' präsentiert wird.

\section{Die (begrenzte) Erweiterung der Kompetenzen}

Der Vertrag von Amsterdam hatte 1999 eine massive Ausweitung der Handlungsbefugnisse von Gemeinschaft und Union in den Bereichen Inneres und Justiz gebracht, die teilweise noch heute nicht voll ausgeschöpft sind. Von einigen Teilbereichen abgesehen - das Fehlen einer ausdrücklichen Handlungsbefugnis im Bereich des Strafverfahrensrechts ist das vornehmlichste Beispiel - haben Kompetenzfragen in der Diskussion der Defizite des RFSR im Vorfeld der Vertragsverhandlungen denn auch eine vergleichsweise geringe

17 Hierzu mehr im Abschnitt „Differenzierung“.

18 Eine der wenigen Veränderungen, die der Vertrag von Lissabon im Bereich des RFSR gegenüber dem Entwurf des Verfassungsvertrages bringt. 
Rolle gespielt. Interessanterweise hat sogar die Kommission in ihren Fortschrittsberichten und -kritik zum Haager Programm ${ }^{19}$ mangelnde rechtliche Handlungsbefugnisse nicht als ein primäres Problem definiert. Dennoch beinhaltet der Vertrag von Lissabon eine Reihe von ausdrücklichen, neuen Formulierungen der Handlungsbefugnisse der Union, die insgesamt eine Kompetenzerweiterung bedeuten, in den einzelnen Politikfeldern recht unterschiedlich ausfallen und auch neuen Grenzen unterworfen werden:

Im Bereich der Außengrenzkontrollen führt der Vertrag erstmals das Ziel eines ,integrierten Grenzschutzsystems“ (Artikel 77(1)(c) AEUV) ein. Hierbei handelt es sich um einen Begriff der seit dem Aktionsplan des Rates für den Grenzschutz an den Außengrenzen vom 13. Juni $2002^{20}$ zum festen Bestandteil der EU-Grenzschutzpolitik gehört und den Aufbau gemeinsamer institutioneller Strukturen (Frontex), die Förderung gemeinsamer Operationen und Interoperabilität der nationalen Grenzschutzbehörden und eine Reihe von Maßnahmen in den Bereichen Ausbildung und Standardisierung umfasst. Durch die Einführung dieses neuen vielgestaltigen Oberbegriffs im Vertrag wird die Rechtsgrundlage für Maßnahmen im Bereich des Außengrenzschutzes materiell erweitert, doch handelt es sich hier in erster Linie um eine Anpassung des vertraglichen Rahmens an einen breiteren Ansatz der Außengrenzschutzpolitik der Union, der im Haager Programm und anderen Schlüsseldokumenten bereits seit Längerem zur Anwendung kommt.

Im Bereich Asyl führt der Vertrag erstmals den Begriff einer ,gemeinsamen Politik im Bereich Asyl, subsidiärer Schutz und vorübergehender Schutz" (Artikel 78(1) AEUV) ein. Materiell gestärkt werden die Handlungsbefugnisse der Union durch die vorgesehene Einführung eines in der ganzen Union gültigen einheitlichen Asylstatus und einen einheitlichen subsidiären Schutzstatus für Drittstaatsangehörige, die keinen europäischen Asylstatus erhalten, aber internationalen Schutz benötigen (Artikel 78(2)(a) und (b) AEUV). Gegenüber den gegenwärtigen Vertragsbestimmungen, die nur gemeinsame Mindeststandards im Bereich der Asylpolitik vorsehen, markiert das Handlungsziel des ,einheitlichen Status“ den Übergang zu einem umfassenderen Ansatz, der Möglichkeiten zur Füllung der bestehenden gesetzgeberischen Lücken und zu einer Konsolidierung der bisher noch recht fragmentierten EU-Asylgesetzgebung bietet und als solche auch vom UN-Hochkommissariat jüngst ausdrücklich begrüßt wurde. ${ }^{21}$ Das Ziel einer gemeinsamen Asylregelung mit einheitlichem Status ist allerdings auch bereits im Haager Programm für die (verzögerte) zweite Asylgesetzgebungsphase vorgesehen, sodass der Vertrag auch hier die Handlungsbefugnisse bereits formulierten Ambitionen anpasst. ${ }^{22}$

Der Begriff ,gemeinsame Politik“ wird durch den Vertrag auch für den Bereich Einwanderung eingeführt. Hier finden sich die vielleicht ambitioniertesten Zielformulierungen des gesamten RFSR, soll diese ,gemeinsame Einwanderungspolitik“ doch ,in allen Phasen eine wirksame Steuerung der Migrationsströme, eine angemessene Behandlung von Drittstaatsangehörigen, die sich rechtmäßig in einem Mitgliedstaat aufhalten, sowie die Verhütung und

19 Siehe Europäische Kommission: Umsetzung des Haager Programms: Weitere Schritte, 2006; Bericht über die Umsetzung des Haager Programms im Jahr 2006, 2007; und Bericht über die Umsetzung des Haager Programms im Jahr 2007, 2008.

20 Rat der Europäischen Union: Plan für den Grenzschutz an den Außengrenzen der Mitgliedstaaten der Europäischen Union, Dok. 10019/02 vom 14. Juni 2002.

21 UNHCR: Building a Europe of Asylum: UNHCR's Recommendations to France for its European Union Presidency (July - December 2008), Brüssel, 9. Juni 2008, S. 1 und 6.

22 Siehe Haager Programm zur Stärkung von Freiheit, Sicherheit und Recht in der Europäischen Union, in: Amtsblatt der EU, Nr. C 53 vom 3. März 2005, Abschnitt 1.3. Zu erwähnen ist hier, dass das Haager Programm 2004 in der Annahme des Inkrafttretens des Verfassungsvertrages formuliert wurde, in dem das Ziel eines einheitlichen Status bereits kodifiziert war. 
verstärkte Bekämpfung von illegaler Einwanderung und Menschenhandel gewährleisten“ (Artikel 79(1) AEUV). Wenn man - überwältigt von dieser beeindruckenden Willenserklärung - dann aber die folgenden Vertragsbestimmungen liest, so wird deutlich, dass die Handlungsbefugnisse im Wesentlichen identisch mit den bisherigen sind und nur teilweise näher definiert werden: So werden in Artikel 79(2)(c) und 79(2)(d) AEUV die schon bisher möglichen Maßnahmen gegen illegale Einwanderung ausdrücklich auf solche gegen ,,illegalen Aufenthalt, einschließlich Abschiebung und Rückführung solcher Personen, die sich illegal in einem Mitgliedstaat aufhalten“ und auf solche zur Bekämpfung des Menschenhandels ausgedehnt, die auch bereits auf den allgemeiner formulierten gegenwärtigen Artikel 63(3)(b) EGV gestützt werden können. Gleiches gilt für die neue ausdrückliche Befugnis des Artikels 79(3) AEUV, mit Drittländern Übereinkünfte über eine Rückübernahme von Einreise- oder Aufenthaltsbedingungen in Mitgliedstaaten nicht oder nicht mehr erfüllenden Drittstaatsangehörigen in ihr Ursprungs- oder Herkunftsland zu schließen, ein Feld, in dem die Union seit Beginn des Jahrzehnts bereits durch die Aushandlung zahlreicher Rücknahme-Übereinkommen - so etwa mit der Russischen Föderation und den Westlichen Balkanländern - beträchtliche Fortschritte erzielt hat. Artikel 79(4) AEUV sieht erstmals Maßnahmen zur Förderung der Integration von in Mitgliedstaaten rechtmäßig ansässigen Drittstaatsangehörigen vor - ein erst in den letzten Jahren vor allem aufgrund der Bemühungen der Kommission aufgewertetes Aktionsfeld. Die Handlungsmöglichkeiten der Union werden jedoch gleich zweifach limitiert, darf diese in diesem Bereich doch nur die „Bemühungen“ der Mitgliedstaaten „fördern und unterstützen“" und dies dann auch noch unter „Ausschluss jeglicher Harmonisierung der Rechtsvorschriften der Mitgliedstaaten“, sodass von einem Kompetenzdurchbruch der Union im Bereich der Integrationspolitik, wo die Mitgliedstaaten immer noch teilweise sehr unterschiedliche Ansätze verfolgen, keinesfalls die Rede sein kann. Die mit Abstand einschneidendste Beschränkung für die neue ,gemeinsame Einwanderungspolitik" findet sich allerdings in Artikel 79(5) AEUV, der den Mitgliedstaaten ausdrücklich das Recht vorbehält, festzulegen, wie viele Drittstaatsangehörige aus Drittländern in ihr Hoheitsgebiet einreisen dürfen, um dort als Arbeitnehmer oder Selbstständige Arbeit zu suchen. Angesichts der innen- und arbeitsmarktpolitischen Sensibilität der Zuwanderung zu Arbeitszwecken ist dieser Vorbehalt ohne Weiteres erklärlich. Dennoch stellt sich die Frage, wie gehaltvoll eine ,gemeinsame Einwanderungspolitik“ der Union sein kann, solange die Mitgliedstaaten nach wie vor rein nationale Politiken im Bereich der Zuwanderung zu Arbeitszwecken verfolgen und folglich auch die, ob man im Hinblick darauf nicht besser auf die hochtrabende Begrifflichkeit des Artikels 79(1) AEUV verzichtet hätte. Man muss nur an die „Gemeinsame“ Außen- und Sicherheitspolitik denken, um sich zu erinnern, dass es der Union bereits jetzt nicht an ambitionierter Terminologie fehlt, bei der Anspruch und Wirklichkeit deutlich auseinanderklaffen und daher kaum zur Glaubwürdigkeit unter den Bürgern beiträgt.

Von beträchtlicher Bedeutung für die Außengrenz-, Asyl- und Einwanderungspolitik zusammengenommen ist die starke neue Betonung des Prinzips der Solidarität der Mitgliedstaaten: Artikel 67(2) AEUV stellt ausdrücklich fest, dass sich die gemeinsamen Politiken der Mitgliedstaaten in diesen Bereichen auf diese Solidarität gründet, und gemäß Artikel 80 AEUV gilt für diese Politikbereiche ,,der Grundsatz der Solidarität und der gerechten Aufteilung der Verantwortlichkeiten unter den Mitgliedstaaten, einschließlich in finanzieller Hinsicht“" mit der Folge, dass erlassene Rechtsakte der Union ,immer wenn dies erforderlich ist, entsprechende Maßnahmen für die Anwendung dieses Grundsatzes“ enthalten sollen. Damit wird erstmals auch im Vertrag anerkannt, dass den Mitgliedstaaten aus den gemeinsamen Verantwortlichkeiten in diesen Bereichen unterschiedliche Lasten entstehen, die beson- 
ders offenkundig im Falle der unterschiedlichen Längen der von den jeweiligen Mitgliedstaaten gemäß der gemeinsamen Schengen-Standards zu überwachenden Außenlandbeziehungsweise Außenseegrenzen sind, aber sich natürlich auch aus den nach wie vor sehr unterschiedlich hohen Zahlen von Asylbewerbern und illegalen Einwanderern ergeben. Mit dem ausdrücklichen Hinweis auf die finanzielle Dimension wird eine deutlich verstärkte Rechtsgrundlage für finanzielle Instrumente zur Unterstützung besonders , exponierter ‘ Mitgliedstaaten - dies gilt etwa für die neuen Mitgliedstaaten mit längeren Außenlandgrenzen sowie zu Finanzhilfen auch im Asyl- und Einwanderungsbereich geschaffen. Artikel 78(3) AEUV sieht ausdrücklich spezifische Unterstützungsmaßnahmen für Mitgliedstaaten vor, die durch einen ,plötzlichen Zustrom von Drittstaatsangehörigen“ in eine Notlage geraten. Da mit jeder Weiterentwicklung der Außengrenz-, Asyl- und Einwanderungspolitik auch die Verpflichtungen der Mitgliedstaaten (Kontrollstandards, notwendige Infrastruktur, Verfahrensgarantien, Ausbildung usw.) steigen, diese aber zugleich auch weiterhin aus geographischen und anderen Gründen unterschiedlichen Belastungen ausgesetzt sein werden, ist die Entwicklung geeigneter Solidaritätsinstrumente eine Schlüsselfrage für die zukünftige Handlungsfähigkeit der Union in diesen Bereichen. Die starke Betonung des Solidaritätsprinzips durch den Vertrag von Lissabon bedeutet daher einen notwendigen und überfälligen Fortschritt.

Im Bereich der justiziellen Zusammenarbeit in Strafsachen werden die Handlungsbefugnisse der Union in Teilbereichen gestärkt: Erstmals ausdrücklich erwähnt werden Maßnahmen zur Sicherstellung der Zusammenarbeit bei der Erhebung von Beweismitteln (Artikel 81(2)(d) AEUV), zur Sicherstellung eines effektiven Zugangs zum Recht (Artikel 81(2)(e) AEUV) ${ }^{23}$ zur Entwicklung von alternativen Methoden für die Beilegung von Streitigkeiten (Artikel 81(2)(g) AEUV) und solche zur Förderung der Weiterbildung von Richtern und Justizbediensteten (Artikel 81(2)(h) AEUV). In allen diesen Bereichen ist die Gemeinschaft zwar bereits auf der Grundlage der gegenwärtigen Vertragsbestimmungen tätig geworden, ${ }^{24}$ aber die nunmehr präzise formulierten Handlungsermächtigungen, die zugleich Zieldefinitionen sind, können die Hand der Kommission bei neuen Gesetzgebungsvorschlägen stärken und möglichen ultra vires Einwänden seitens einzelner Mitgliedstaaten zuvorkommen. Eine Neuerung ist auch die nunmehr ausdrückliche Erwähnung der Möglichkeit von Maßnahmen zur Angleichung der Rechtsvorschriften der Mitgliedstaaten, eine explizite Befugnis zu Harmonisierungsmaßnahmen im Bereich des Zivilrechts, die in den Verhandlungen über den Vertrag von Amsterdam noch höchst umstritten war.

Die Erweiterung der Kompetenzen der Union in den jetzigen Bereichen der ,dritten Säule" gehörten zu den schwierigsten Verhandlungspunkten hinsichtlich des RFSR. Das Weiterbestehen einer Reihe von ,Inseln der Einstimmigkeit‘ und die Einführung des ,Notbremsenverfahrens' (siehe oben) zeigen, wie problematisch, zumindest einigen Mitglied-

23 Die Handlungsermächtigung der Union im Bereich des Zugangs zum Recht wird zusätzlich durch Artikel 67(4) AEUV gestärkt, dem zufolge die Union den „Zugang zum Recht“ erleichtert, insbesondere durch den „Grundsatz der gegenseitigen Anerkennung gerichtlicher und außergerichtlicher Entscheidungen in Zivilsachen".

24 Beispiele sind im Bereich der Beweismittel die Verordnung (EG) 1206/2001 des Rates vom 28. Mai 2001 über die Zusammenarbeit zwischen den Gerichten der Mitgliedstaaten auf dem Gebiet der Beweisaufnahme in Zivil- oder Handelssachen, in: Amtsblatt der EG, Nr. L 174 vom 27. Juni 2001; und im Bereich des Zugangs zum Recht die Richtlinie 2002/8/EG des Rates vom 27. Januar 2003 zur Verbesserung des Zugangs zum Recht bei Streitsachen mit grenzüberschreitendem Bezug durch Festlegung gemeinsamer Mindestvorschriften für die Prozesskostenhilfe, in: Amtsblatt der EG, Nr. L 26 vom 31. Januar 2003; und im Bereich der alternativen Streitbeilegungsverfahren die Richtlinie 2008/52/EG des Europäischen Parlaments und des Rates vom 21. Mai 2008 über bestimmte Aspekte der Mediation in Zivil- und Handelssachen, in: Amtsblatt der EU, Nr. L 136 vom 24. Mai 2008. 
staaten, eine mögliche stärkere Rolle der Union in den Bereichen der polizeilichen und der justiziellen Zusammenarbeit in Strafsachen noch immer erscheint. Dennoch sieht der Vertrag einige beträchtliche Erweiterungen der Handlungsmöglichkeiten auf Unionsebene vor:

Im Bereich der justiziellen Zusammenarbeit in Strafsachen wird das Prinzip der gegenseitigen Anerkennung gerichtlicher Urteile und Entscheidungen nun formell zum „Grundsatz" dieser Zusammenarbeit erhoben (Artikel 82(1) AEUV), was nicht nur eine nachträglichen Kodifizierung der bereits vom Europäischen Rat in Tampere vom Oktober 1999 diesem Prinzip zugewiesenen Priorität bedeutet, sondern auch eine Verbreiterung der Handlungsgrundlage. Während es sich bei der neuen ausdrücklichen Befugnis, Maßnahmen zur Weiterbildung von Richtern und Staatsanwälten sowie Justizbediensteten zu fördern (Artikel 82(1)(c) AEUV) um eine eher bescheidene - aber im Hinblick auf die praktische Bedeutung solcher Maßnahmen - überfällige Kompetenzergänzung handelt, öffnet der Vertrag der Union ein zumindest teilweise neues - und potenziell weites - Aktionsfeld im Bereich des Strafverfahrensrechts: Erstmals ausdrücklich vorgesehen werden ,zur Erleichterung der gegenseitigen Anerkennung" Mindestvorschriften hinsichtlich der Zulässigkeit von Beweismitteln auf gegenseitiger Basis zwischen den Mitgliedstaaten, der Rechte des Einzelnen im Strafverfahren, der Rechte der Opfer von Straftaten und ,sonstiger spezifischer Aspekte des Strafverfahrens“ (Artikel 82(2)(a)-(d) AEUV). Vor allem durch den Einschluss der ,,sonstigen spezifischen Aspekte" - die allerdings der Einstimmigkeit im Rat unterworfen sind wird damit potenziell der gesamte Bereich des Strafverfahrensrechts für Rechtsangleichungsmaßnahmen der Union eröffnet. Diese Öffnung erscheint insoweit notwendig - und sogar überfällig - als sich in den vergangenen Jahren gezeigt hat, dass die Anwendung und Weiterentwicklung des Prinzips der gegenseitigen Anerkennung von Urteilen und richterlichen Entscheidungen - und das gegenseitige Vertrauen, das diese voraussetzen - sowohl unter Richtern als auch Regierungen und Parlamenten aufgrund der immer noch ausgeprägten Unterschiede in den strafverfahrensrechtlichen Standards der einzelnen Mitgliedstaaten auf deutliche Grenzen stoßen. Ohne Fortschritte bei der Angleichung der strafverfahrensrechtlichen Regelungen steht der so häufig als Kernelement der justiziellen Zusammenarbeit in Strafsachen herausgestellte Grundsatz der gegenseitigen Anerkennung auf wenig tragfähigen Füßen, ${ }^{25}$ sodass der Vertrag mit der aufgezeigten massiven Öffnung nur eine weitere überfällige Anpassung der entsprechenden Kompetenzen der Union vornimmt. Indem Artikel 82(2) AEUV ausdrücklich die betreffenden Mindestvorschriften an die Berücksichtigung ,der Unterschiede zwischen den Rechtsordnungen und -traditionen der Mitgliedstaaten" bindet, wird aber auch der Tatsache Rechnung getragen, dass es sich beim Strafrecht um einen stark von teilweise divergierenden Rechtstraditionen und -prinzipien geprägten Kernbereich einzelstaatlicher Souveränität handelt, und derselbe Paragraph belässt den Mitgliedstaaten auch die Möglichkeit, ein höheres strafverfahrensrechtliches Schutzniveau für den Einzelnen beizubehalten oder einzuführen. Die nach mehrjährigen Bemühungen im Juni 2007 im Rat gescheiterten Verhandlungen über einen Rahmenbeschluss über bestimmte Verfahrensrechte in Strafverfahren innerhalb der Europäischen Union ${ }^{26}$ haben gezeigt, dass Harmonisierungsmaßnahmen in diesem Bereich ein erhebliches Risiko von Einigungen auf den kleinsten gemeinsamen Nenner mit sich bringen, sodass die Möglichkeit der Beibehaltung höherer Standards ein notwendiges Korrektiv darstellt. Wie oben erwähnt, kann im Be-

25 Siehe hierzu im weiteren Zusammenhang Valsamis Mitsilegas: The Transformation of Criminal Law in the ,Area of Freedom, Security and Justice', in: Yearbook of European Law 2007, Oxford 2008, S. 1-32.

26 Siehe den ursprünglichen Kommissionsvorschlag Europäische Kommission: Vorschlag für einen Rahmenbeschluss des Rates über bestimmte Verfahrensrechte in Strafverfahren innerhalb der Europäischen Union, $\operatorname{KOM}(2004) 328$. 
reich des Strafverfahrensrechts für alle nicht unter die Kategorie der „sonstigen“ - und folglich ohnehin durch Einstimmigkeit ,geschützte' Aspekte - fallende Maßnahmen durch jeden einzelnen Mitgliedstaat gemäß Artikel 82(3) AEUV die sogenannte ,Notbremse ' betätigt werden, auch dies ein deutlicher Hinweis auf die Sensibilität dieses Bereichs - und ein nicht geringer Preis für den Übergang zu Mehrheitsabstimmungen.

Kompetenzerweiterungen sieht der Vertrag auch im Bereich der Harmonisierung des materiellen Strafrechts vor. Bislang beschränkt Artikel 31(e) EUV die Handlungsbefugnis der Union auf Mindestvorschriften zur Festlegung von Straftaten und Strafen auf nur drei Bereiche schwerwiegender grenzüberschreitender Kriminalität: organisierte Kriminalität, Terrorismus und illegaler Drogenhandel. In Artikel 83(1) Absatz 2 AEUV wird diese Harmonisierungsbefugnis auf sieben weitere Felder ausgeweitet: Menschenhandel, sexuelle Ausbeutung von Frauen und Kindern, illegaler Waffenhandel, Geldwäsche, Korruption, Fälschung von Zahlungsmitteln und Computerkriminalität. Da der bisherige Begriff der organisierten Kriminalität bereits zumindest teilweise Aspekte aller dieser Kriminalitätsformen mit abdeckt, ist diese Kompetenzerweiterung weniger extensiv, als die stark verlängerte Liste dies erscheinen lassen kann, und tatsächlich ist die Union in vielen dieser Bereiche bereits gesetzgeberisch tätig geworden. ${ }^{27}$ Dennoch könnte die nunmehr präzise ausformulierte Rechtsgrundlage in einigen bislang eher unterentwickelten Bereichen - so etwa der sexuellen Ausbeutung von Frauen und Kindern, der Korruption und der Computerkriminalität die Handlungsfähigkeit der Union deutlich stärken. Dies gilt umso mehr als der Rat hierüber nunmehr auch mit qualifizierter Mehrheit entscheidet, ein Reformschritt, der allerdings um den Preis einer weiteren ,Notbremse' erkauft wurde.

Von größerer Bedeutung als die Erweiterung der genannten einzelnen Kriminalitätsbereiche ist die in Artikel 83(1) Absatz 3 AEUV eröffnete Möglichkeit zum Erlass eines Ratsbeschlusses, in dem ,,andere Kriminalitätsbereiche“ bestimmt werden, die dem Kriterium ,besonders schwerer Kriminalität“" genügen, die gemäß Artikel 83(1) Absatz 1 AEUV ,aufgrund der Art oder der Auswirkungen der Straftaten oder aufgrund einer besonderen Notwendigkeit, sie auf einer gemeinsamen Grundlage zu bekämpfen, eine grenzüberschreitende Dimension haben". Artikel 83(2) AEUV etabliert zusätzlich eine Handlungsbefugnis zu Harmonisierungsmaßnahmen im Bereich des materiellen Strafrechts für Materien, die Gegenstand von Harmonisierungsmaßnahmen in anderen Politikfeldern der Union geworden sind, falls dies für „,die wirksame Durchführung der Politik der Union [unerlässlich]“ ist. ${ }^{28}$ Artikel 83(1) und (2) AEUV zusammengenommen eröffnen zumindest potenziell weite Teile des materiellen Strafrechts der Mitgliedstaaten für eventuelle EU-Harmonisierungsbemühungen, eine Perspektive, die nicht nur im Vereinigten Königreich Unbehagen ausgelöst hat. Der Übergang zu ,,anderen Kriminalitätsbereichen“ wird daher denn auch von einem einstimmigen Beschluss des Rates abhängig gemacht.

Damit sind die Neuerungen hinsichtlich der Kompetenzen im strafrechtlichen Bereich aber noch nicht erschöpft: Artikel 84 AEUV gewährt der Union erstmals eine Handlungsbefugnis zur „Förderung des Vorgehens der Mitgliedstaaten im Bereich der Kriminalprävention“, doch wird diese materiell stark dadurch beschnitten, dass ,,jegliche Harmonisierung der Rechtsvorschriften der Mitgliedstaaten“ ausdrücklich ausgeschlossen wird. Weitaus bedeutsamer ist demgegenüber die durch Artikel 86(1) AEUV geschaffene Möglichkeit, zur

27 So etwa im Bereich des Menschenhandels durch den Rahmenbeschluss des Rates vom 19. Juli 2002 zur Bekämpfung des Menschenhandels (2002/629 JI), in: Amtsblatt der EG, Nr. L 203 vom 1. August 2002.

28 Ein Beispiel hierfür wären strafrechtliche Maßnahmen zum Zweck des Umweltschutzes, die zu der erwähnten wichtigen Entscheidung des EuGH von 2005 (Europäischer Gerichtshof: Rs. C-176/03 (Kommission/Rat), 2005) geführt haben. 
Bekämpfung von Straftaten zum Nachteil der finanziellen Interessen der Union ,,ausgehend von Eurojust eine Europäische Staatsanwaltschaft" einzusetzen. Diese Staatsanwaltschaft soll gemäß Artikel 86(2) AEUV zuständig sein ,für die strafrechtliche Untersuchung und Verfolgung sowie die Anklageerhebung in Bezug auf Personen, die als Täter oder Teilnehmer Straftaten zum Nachteil der finanziellen Interessen der Union begangen haben“ und „,bei diesen Straftaten vor den zuständigen Gerichten der Mitgliedstaaten die Aufgaben der Staatsanwaltschaft wahr[nehmen]". Gemäß Artikel 86(4) AEUV kann der Europäische Rat - auch hier kommt eine quasi-gesetzgeberische Funktion der Staats- und Regierungschefs zur Anwendung - mit Zustimmung des Europäischen Parlaments und nach Anhörung der Kommission jederzeit einstimmig die Ausdehnung der Befugnisse der Europäischen Staatsanwaltschaft auf die Bekämpfung der schweren Kriminalität mit grenzüberschreitender Dimension hinsichtlich Personen ausweiten, die als Täter oder Teilnehmer schwere, mehr als einen Mitgliedstaat betreffende Straftaten begangen haben. Die Einrichtung einer solchen Institution würde fraglos einen bedeutenden Schritt in Richtung auf einen effektiven europäischen Ermittlungs- und Strafverfolgungsraum mit entsprechenden Effizienzgewinnen bei der Bekämpfung der betreffenden Kriminalitätsformen markieren und - insbesondere aufgrund der grenzüberschreitenden Handlungsbefugnis dieser Staatsanwaltschaft - auch eine bedeutende integrationspolitische Entwicklung darstellen.

Die Hürden für diesen Schritt dürften allerdings trotz der somit geschaffenen Möglichkeit beträchtlich sein. Zum einen ist bereits für die Einsetzung der Staatsanwaltschaft Einstimmigkeit im Rat erforderlich, zu der im Falle der Ausweitung der Befugnisse die erforderliche Einstimmigkeit im Europäischen Rat hinzukommt. Sodann wird aus den Vertragsbestimmungen nicht deutlich, wie diese Staatsanwaltschaft von Eurojust, das gegenwärtig andere Funktionen ausübt, ,ausgehen“ soll. Schließlich - und dies wiegt wohl am schwersten kann eine solche Europäische Staatsanwaltschaft nur dann wirksam und mit der notwendigen Rechtssicherheit agieren, wenn sie auf der Grundlage eines entsprechenden Besitzstandes an materiellem europäischen Strafrecht und Strafverfahrensrecht tätig werden kann. Hierzu würde es zumindest in Ansätzen eines dem US-Bundesstrafrecht mit seinen „federal crimes“ vergleichbaren Rahmens bedürfen, von dem die Union gegenwärtig noch weit entfernt ist.

Für den wahrscheinlichen Fall, dass die Einrichtung der Europäischen Staatsanwaltschaft noch auf sich warten lassen sollte, ist die institutionelle Seite der strafrechtlichen Zusammenarbeit allerdings nicht völlig zum Stillstand verurteilt, da der Vertrag von Lissabon für Eurojust erstmals ausdrücklich die Möglichkeit der „Einleitung“ von Ermittlungsmaßnahmen sowie Vorschläge zur Einleitung von strafrechtlichen Verfolgungsmaßnahmen vorsieht, die jeweils von den zuständigen nationalen Behörden durchgeführt werden (Artikel 85(1)(a) AEUV). Zwar kann Eurojust auch schon gegenwärtig entsprechende Empfehlungen formulieren, ${ }^{29}$ aber bei einer Ausschöpfung des mit dieser neuen Vertragsbestimmung gegebenen Handlungsspielraums dürfte es für die einzelstaatliche Behörden nur noch begrenzte, sehr begrenzte Möglichkeiten geben, einer Initiative von Eurojust nicht zu entsprechen. Zudem wird Eurojust durch Artikel 85(1)(c) AEUV auch eine im Vertrag neue - in der Praxis allerdings bereits existierende - Funktion der Vermittlung bei Kompetenzkonflikten zugewiesen.

Im Vergleich zur strafrechtlichen Zusammenarbeit sieht der Vertrag für die polizeiliche Zusammenarbeit eher begrenzte Erweiterungen der Handlungsmöglichkeiten vor: Artikel 87(2)(c) AEUV sieht nunmehr die Möglichkeit von Maßnahmen zu ,gemeinsamen Ermitt-

29 Auf der Grundlage von Artikel 6 und 7 des Ratsbeschlusses vom 28. Februar 2002 über die Errichtung von Eurojust. 
lungstechniken“ - und nicht mehr nur wie im gegenwärtigen Artikel 30(1)(d) EUV die „Bewertung" solcher Maßnahmen vor, was die Einführung gemeinsamer Mindeststandards begünstigen könnte. Grenzüberschreitende polizeiliche Operationen unterliegen in der Europäischen Union gegenwärtig immer noch der Vielzahl teilweise stark divergierender biund multilateraler Vereinbarungen hinsichtlich der Befugnisse, die Polizeibeamte unter welchen Bedingungen auf dem Gebiet des jeweiligen anderen Mitgliedstaates ausüben können, ein Dschungel unterschiedlicher und oft sehr restriktiver Vorschriften, der solche Operationen oft behindert. Artikel 89 AEUV sieht erstmals vor, dass der Rat - nach Anhörung des Parlaments - festlegt, ,unter welchen Bedingungen und innerhalb welcher Grenzen [Polizeibehörden] [...] der Mitgliedstaaten im Hoheitsgebiet eines anderen Mitgliedstaats in Verbindung und in Absprache mit dessen Behörden tätig werden dürfen“. Hierfür ist allerdings Einstimmigkeit im Rat erforderlich, sodass sich eine gehaltvolle Einigung wahrscheinlich nicht leicht erzielen werden lässt.

Die im gegenwärtigen Artikel 30(2) EUV recht umständliche Beschreibung der Aufgaben von Europol wird durch den Vertrag gestrafft. Artikel 88(2)(a) AEUV schafft eine umfassende Vertragsgrundlage für das „Einholen, Speichern, Verarbeiten, Analysieren und Austauschen von Informationen“ durch Europol, die nunmehr auch ausdrücklich an Behörden von „Drittländern beziehungsweise Stellen außerhalb der Union übermittelt werden” können. Alle diese Aufgaben erfüllt Europol bereits - auf teilweise sehr dünner Rechtsgrundlage -, sodass es sich hier um eine überfällige Ergänzung handelt. Einen Fortschritt für Europol bringt Artikel 88(2)(b) AEUV, dem zufolge die Polizeibehörde mit der „Koordinierung, Organisation und Durchführung von Ermittlungen und von operativen Maßnahmen, die gemeinsam mit den zuständigen Behörden der Mitgliedstaaten oder im Rahmen gemeinsamer Ermittlungsgruppen durchgeführt werden“, beauftragt werden kann. Diese Formulierung geht deutlich über die bisher einzig vorgesehenen Möglichkeiten der Unterstützung von Ermittlungsmaßnahmen der Mitgliedstaaten und von „Ersuchen“ hinsichtlich des Einleitens und der Koordinierung von Ermittlungsmaßnahmen hinaus. Um einen Durchbruch hinsichtlich der Übertragung operativer Befugnisse auf Europol handelt es sich jedoch nicht, da Artikel 88(3) AEUV ausdrücklich vorschreibt, dass Europol ,operative Maßnahmen nur in Verbindung und in Absprache mit den Behörden des Mitgliedstaats oder der Mitgliedstaaten ergreifen [darf], deren Hoheitsgebiet betroffen ist“, und „die Anwendung von Zwangsmaßnahmen [...] ausschließlich den zuständigen einzelstaatlichen Behörden vorbehalten“ bleibt. Der Koordinierungsauftrag, der Europol übertragen werden kann, könnte auch durch den in Artikel 71 AEUV vorgesehenen neuen Ratsausschuss zur Förderung und Verstärkung der „operative Zusammenarbeit im Bereich der inneren Sicherheit“ und zur „Koordinierung der Maßnahmen der zuständigen Behörden der Mitgliedstaaten" eingeschränkt werden. ${ }^{30}$ Obwohl - vor allem im polizeilichen Bereich - kein Zweifel an der Notwendigkeit einer besseren Koordinierung der wachsenden operativen Dimension des RFSR bestehen kann, wären sich überschneidende Koordinierungsbefugnisse größerer Effizienz gewiss nicht dienlich.

Die Europol im operativen Bereich auferlegten Beschränkungen entsprechen einer für den gesamten RFSR bedeutsamen Beschränkung der Unionskompetenzen: Gemäß Artikel 4(2) EUV-Lissabon fällt ,,insbesondere die nationale Sicherheit [...] weiterhin in die alleinige Verantwortung der einzelnen Mitgliedstaaten“, und gemäß Artikel 72 AEUV berühren die Bestimmungen zum RFSR nicht ,die Wahrnehmung der Zuständigkeiten der Mitglied-

30 Dieser ist unter der Bezeichnung „COSI“ („Comité de sécurité intérieure“) bereits zum Gegenstand intensiver Verhandlungen im Rat geworden. 
staaten für die Aufrechterhaltung der öffentlichen Ordnung und den Schutz der inneren Sicherheit". Mit diesen beiden Bestimmungen wird die ausschließliche Zuständigkeit der Mitgliedstaaten für alle Fragen der inneren Sicherheit vertragsrechtlich noch deutlicher als bisher geschützt, woraus sich erhebliche Einspruchsmöglichkeiten einzelner Mitgliedstaaten etwa gegen etwaige weitergehende Initiativen ergeben könnten. Diese Beschränkungen entsprechen weitgehend der restriktiven Logik des ersten Aktionsplans zur Entwicklung des RFSR vom Dezember 1998, ${ }^{31}$ sodass sich an den Vorbehalten zumindest einiger Mitgliedstaaten trotz der Entwicklung von zehn Jahren offenkundig wenig geändert hat.

\section{Die (retardierte) Stärkung der Umsetzungsfähigkeit}

Auch die umfassendste Erweiterung von Entscheidungsfähigkeit und Kompetenzen bedeutet noch keine entsprechende Verstärkung der Handlungsfähigkeit, wenn nicht auch eine wirksame Umsetzung angenommener Entscheidungen gewährleistet werden kann. Die Kommission hat zu Recht auf den Zusammenhang zwischen den gegenwärtigen erheblichen Umsetzungsdefiziten von EU-Rechtsakten im Bereich der ,dritten Säule durch die Mitgliedstaaten - mit teilweise mehrjährigen Verzögerungen bei Rahmenbeschlüssen - und der fehlenden Möglichkeit eines dem gegenwärtigen Artikel 226 EGV entsprechenden Vertragsverletzungsverfahrens verwiesen. ${ }^{32}$ Mit der Aufhebung der ,Säulenteilung ' und der sich daraus ergebenden, Vergemeinschaftung ' des Titels VI EUV ist folgerichtig auch eine Ausdehnung sämtlicher Verfahrensmöglichkeiten vor dem Gerichtshof auf die Bereiche der ,dritten Säule“ verbunden. Gemäß Artikel 10 des dem Vertrag von Lissabon beigefügten „Protokolls über die Übergangsbestimmungen“33 gelten allerdings für eine Übergangsperiode von fünf Jahren die Befugnisse der Kommission zur Einleitung von Vertragsverletzungsverfahren (Artikel 258 AEUV) nicht für die vor Inkrafttreten des neuen Vertrages in den Bereichen der, dritten Säule' angenommenen Rechtsakte, sodass das bestehende ,zwangsfreie‘ Umsetzungsregime für den betreffenden Besitzstand temporär erhalten bleibt. Ganz abgesehen von ihren negativen Auswirkungen für die Umsetzungsdisziplin unter den Mitgliedstaaten, kann diese Ausnahmeregelung - ebenso wie die fragwürdigen Kapriolen bei den Entscheidungsverfahren - als Indiz dafür genommen werden, wie wenig den ,Vätern der Verträge" mittlerweile noch eine einheitliche Rechts- und Verfahrensordnung der Union gilt.

Eine - in diesem Fall nicht retardierte - Neuerung zur Stärkung der Umsetzungsfähigkeit sieht Artikel 70 AEUV vor, dem zufolge Maßnahmen zur ,unparteiischen Bewertung“ der Durchführung der den RFSR betreffenden Unionspolitik durch die Behörden der Mitgliedstaaten ergriffen werden können, ,insbesondere um die umfassende Anwendung des Grundsatzes der gegenseitigen Anerkennung zu fördern“. Hierbei handelt es sich um die vertragliche Kodifizierung der vor allem in den Bereichen des Schengen-Besitzstandes, der Terrorismusbekämpfung und der Erweiterung bereits bestehenden Praxis der gegenseitigen

31 Im sogenannten „Wiener Aktionsplan“ war betont worden, dass es nicht die Absicht des RFSR sei, ,,einen Europäischen Raum der Sicherheit zu schaffen, in dem alle Strafverfolgungsbehörden in Europa in Sicherheitsfragen einheitliche Ermittlungs- und Fahndungsverfahren anwenden“ und dass „,die jeweiligen Kompetenzen der Mitgliedstaaten zur Wahrung der öffentlichen Ordnung und zur Gewährleistung der inneren Sicherheit“ keinesfalls berührt werden dürften (Aktionsplan des Rates und der Kommission zur bestmöglichen Umsetzung der Bestimmungen des Amsterdamer Vertrags über den Aufbau eines Raums der Freiheit, der Sicherheit und des Rechts, in: Amtsblatt der EG, Nr. C 19 vom 23. Januar 1999, Paragraph 10).

32 Siehe Europäische Kommission: Umsetzung des Haager Programms: Weitere Schritte, 2006, S. 12 und 14; und Bericht über die Umsetzung des Haager Programms im Jahr 2007, 2008, S. 16-17.

33 Protokoll (Nr. 36): Über die Übergangsbestimmungen, in: Amtsblatt der EU, Nr. C 115 vom 9. Mai 2008, S. 322-326. 
beziehungsweise kollektiven Evaluierung der Umsetzung von Maßnahmen auf der einzelstaatlichen Ebene. Die neue ausdrückliche Vertragsgrundlage könnte eine Ausweitung und Verbesserung dieser Verfahren fördern, die aufgrund ihrer über die gesetzgeberische Umsetzung hinausgehende Konzentration auf Aspekte der praktischen Umsetzung durch die einzelstaatlichen Behörden - die von Vertragsverletzungsverfahren zumeist gar nicht oder nur zum Teil erfasst werden können - von einigem Nutzen sind.

\section{Verstärkte Kontrolle}

Dem Unionsbürger sollen durch den RFSR zwar gewisse Vorteile geboten werden - wie ein „hohes Maß an Sicherheit“ und ein erleichterter „Zugang zum Recht“ (Artikel 67(3) und (4) AEUV) -, aber er kann auch potenziell zu einem seiner Opfer werden. Maßnahmen im Bereich des RFSR können Rechte von Einzelnen in sehr viel unmittelbarerer Form berühren, als beispielsweise wirtschaftliche Regelungen im Bereich des Binnenmarkts. Der RFSR umfasst in seinem potenziellen Maßnahmenbereich immerhin einige der stärksten Formen staatlicher Machtausübung gegenüber dem Einzelnen, wie etwa Verhaftung, Gefängnisstrafen, das Einfrieren von Vermögenswerten und die Speicherung und Auswertung von persönlichen Daten. Dies gilt auch für Drittstaatsangehörige, die - als Asylsuchende oder illegale Einwanderer - der restriktiven Seite der EU-Asyl- und Einwanderungspolitik (reduzierte Verfahrensgarantien bei „offensichtlich unbegründeten Asylanträgen“, Rückführungsabkommen mit bestimmten Drittstaaten usw.) ausgesetzt sein können. Von daher sind wirksame parlamentarische und richterliche Kontrollverfahren für diesen Bereich der Unionspolitik von besonderer Bedeutung. Der Vertrag von Lissabon bringt in dieser Hinsicht erhebliche Fortschritte:

Durch die Einführung des ,ordentlichen Gesetzgebungsverfahrens“ als Standardverfahren, auch für die polizeiliche und justizielle Zusammenarbeit in Strafsachen, erhält das Europäische Parlament (EP) erstmals ein volles Mitbestimmungsrecht für Rechtsinstrumente in den Bereichen der ,dritten Säule‘. Der Vertrag entspricht damit einer seit Langem erhobenen Forderung des Parlaments - und einem zur demokratischen Legitimation der sich in diesen Bereichen stark ausweitenden Aktion der Union überfälligen Reformbedarf. Für eine Reihe von Materien bleiben teilweise deutlich weniger umfassende Beteiligungsformen des Parlaments erhalten: die Verwaltungszusammenarbeit zwischen den Dienststellen der Mitgliedstaaten im Bereich des RFSR (Artikel 74 AEUV: Anhörung des EP); für die Ausübung der Freizügigkeit relevante Bestimmungen betreffend Pässe, Personalausweise, Aufenthaltstitel oder diesen gleichgestellte Dokumente (Artikel 77(3) AEUV: Anhörung des EP); Maßnahmen zugunsten von Mitgliedstaaten, die von einem plötzlichen Zustrom von Drittstaatsangehörigen betroffen sind (Artikel 78(3) AEUV: Anhörung des EP); Maßnahmen zum Familienrecht mit grenzüberschreitendem Bezug (Artikel 81(3) AEUV: Anhörung des EP); Mindestvorschriften für „sonstige“ (d.h. nicht bereits ausdrücklich vorgesehene) spezifische Aspekte des Strafverfahrensrechts (Artikel 82(2)(d) AEUV: Zustimmung des EP); die Bestimmung ,anderer“ (d.h. nicht bereits ausdrücklich erwähnter) Bereiche schwerwiegender Kriminalität, für die der Rat Mindestvorschriften zur Festlegung von Straftaten und Strafen erlassen kann (Artikel 83(1) AEUV: Zustimmung des EP); die Einsetzung einer Europäischen Staatsanwaltschaft sowie die Ausweitung von deren Zuständigkeiten (Artikel 86(1) und (3) AEUV: Zustimmung des EP); Maßnahmen zur operativen Zusammenarbeit zwischen den Polizeibehörden der Mitgliedstaaten (Artikel 87(3) AEUV: Anhörung des EP) und die Festlegung der Bedingungen und Grenzen innerhalb derer Polizeibehörden der Mitgliedstaaten im Hoheitsgebiet eines anderen Mitgliedstaats in Verbindung und in Absprache mit dessen Behörden tätig werden dürfen (Artikel 89 AEUV: Anhörung des EP). Dennoch aber versetzt der Vertrag von Lissabon 
das Europäische Parlament in eine im Vergleich zur jetzigen Situation ungleich bessere Lage, die Entwicklung des RFSR insgesamt politisch besser kontrollieren und aktiv mitgestalten zu können. Angesichts der häufig scharfen - und teilweise auch recht ideologisch formulierten Kritik bisheriger Rechtsakte der , dritten Säule` durch das Parlament, gibt es auf Seiten der nationalen Ministerien gewisse Befürchtungen, dass die neuen Mitentscheidungsrechte des Parlaments zukünftige Fortschritte in diesen Bereichen erheblich erschweren könnten. ${ }^{34}$ Es kann jedoch wohl davon ausgegangen werden, dass ein durch die „Mitentscheidung“ nunmehr auch der Mitverantwortung unterworfenes Parlament, nicht zuletzt auch aufgrund der Sicherheitserwartungen der Unionsbürger, zu einer pragmatischeren Abwägung des Für und Wider bestimmter gesetzgeberischer Maßnahmen in diesen Bereichen gelangen wird.

Eine gänzlich neue Dimension parlamentarischer Kontrolle wird in den RFSR durch Artikel 69 AEUV eingeführt. Dieser sieht vor, dass die nationalen Parlamente bei Gesetzgebungsvorschlägen und -initiativen im Bereich der polizeilichen und der justiziellen Zusammenarbeit in Strafsachen „Sorge für die Achtung des Subsidiaritätsprinzips“ nach Maßgabe des Protokolls (Nr. 2) über die „Anwendung der Grundsätze der Subsidiarität und der Verhältnismäßigkeit“ ${ }^{35}$ tragen. Gemäß Artikel 7(2) des genannten Protokolls können demnach im Falle einer Mindestzahl von „begründeten Stellungnahmen“ seitens einzelstaatlicher Kammern, ,wonach der Entwurf eines Gesetzgebungsakts nicht mit dem Subsidiaritätsprinzip im Einklang steht“, eine Überprüfung des betreffenden Gesetzgebungsakts erzwungen werden. Diese Mindestzahl liegt bei Maßnahmen im Bereich der polizeilichen und strafrechtlichen Zusammenarbeit bei einem Viertel der Stimmen aller Kammern, in allen anderen Bereichen bei einem Drittel - eine weitere Spur der aufgehobenen ,dritten Säule‘. Da durch das Protokoll (Nr. 1) über die „Rolle der nationalen Parlamente“ ${ }^{\text {“36 }}$ zugleich auch die Informationslage der nationalen Parlamente deutlich verbessert wird, bedeutet dies eine wirkliche Interventionsmöglichkeit der nationalen Parlamente im Legislativprozess der Union. Die wirksame Nutzung dieser Möglichkeit, die zu völlig neuen Konfliktkonstellationen im Rahmen des Gesetzgebungsprozesses führen könnte, würde allerdings eine deutlich verbesserte Zusammenarbeit und Koordination der nationalen Kammern voraussetzen.

Hinsichtlich der richterlichen Kontrollmöglichkeiten hebt der Vertrag von Lissabon alle gegenwärtig noch bestehenden Beschränkungen der Rolle des in „Gerichtshof der Europäischen Union“ umbenannten EuGH auf, dies allerdings mit zwei Ausnahmen: Für die bereits erwähnte Übergangsperiode von fünf Jahren nach Inkrafttreten des Vertrages sind auf die vor Inkrafttreten des neuen Vertrages angenommenen Rechtsakte in den Bereichen der polizeilichen und justiziellen Zusammenarbeit in Strafsachen nicht nur die Befugnisse der Kommission zur Einleitung von Vertragsverletzungsverfahren nicht anwendbar, sondern auch nicht die durch den neuen Vertrag erweiterten Befugnisse des Gerichtshofs. Der gegenwärtige Besitzstand der ,dritten Säule ' wird somit gegen die erweiterte richterliche Kontrolle für die Übergangsperiode noch , geschützt'. Die zweite Ausnahme besteht in der Beibehaltung der besonderen Ordre-Public-Klausel für die polizeiliche und die justizielle Zusammenarbeit in Strafsachen: Gemäß Artikel 276 AEUV ist der Gerichtshof in diesen Bereichen ausdrücklich ,nicht zuständig für die Überprüfung der Gültigkeit oder Verhältnismäßigkeit von Maßnahmen der Polizei oder anderer Strafverfolgungsbehörden eines Mitgliedstaats oder der Wahrnehmung der Zuständig-

34 Interview-Informationen, Generalsekretariat des Rates der Union, Bundesinnenministerium und französisches Innenministerium.

35 Protokoll (Nr. 2): Über die Anwendung der Grundsätze der Subsidiarität und der Verhältnismäßigkeit, in: Amtsblatt der EU, Nr. C 115 vom 9. Mai 2008, S. 206-209.

36 Protokoll (Nr. 1): Über die Rolle der nationalen Parlamente in der Europäischen Union, in: Amtsblatt der EU, Nr. C 115 vom 9. Mai 2008, S. 203-205. 
keiten der Mitgliedstaaten für die Aufrechterhaltung der öffentlichen Ordnung und den Schutz der inneren Sicherheit“. Diese Klausel ist identisch mit dem gegenwärtigen Artikel 35(5) EUV und entspricht der durch Artikel 4(2) EUV-Lissabon und Artikel 72 AEUV geschützten ausschließlichen Kompetenz der Mitgliedstaaten in Fragen der einzelstaatlichen Sicherheit.

Vom Gesichtspunkt eines einheitlichen und umfassenden Rechtsschutzes mag man beide Ausnahmen bedauern, doch nehmen sich diese vor dem Hintergrund der generellen Stärkung der Rolle des Gerichtshofs eher gering aus: Als Folge der Aufhebung der bestehenden Restriktionen wird das Vorabentscheidungsverfahren auch in den Bereichen der ,dritten Säule` - wo es gegenwärtig von nur 16 Mitgliedstaaten , freiwillig ' akzeptiert wird - für alle Mitgliedstaaten verbindlich. Zugleich entfällt gemäß Artikel 267 AEUV die gegenwärtig auf die Materien der ,ersten Säule“ im Bereich des RFSR anwendbare Beschränkung der Einleitung von Vorabentscheidungsverfahren auf letztinstanzliche nationale Gerichte sowie auch der auf die ,dritte Säule " anwendbare Ausschluss von Entscheidungen des Gerichtshofes zu primärrechtlichen Fragen. Damit werden sowohl die Kohärenz der EU-Rechtsordnung als auch die Schutzmöglichkeiten der Rechte des Einzelnen im Bereich des RFSR gestärkt. Noch bedeutsamer für letztere ist die durch Artikel 263 Absatz 4 AEUV erweiterte und nunmehr auf alle Bereiche des RFSR ausgedehnte Möglichkeit der Einleitung von Nichtigkeitsklagen durch natürliche oder juristische Person gegen an sie gerichtete oder sie unmittelbar und individuell betreffende Handlungen der Einrichtungen der Union sowie gegen Rechtsakte mit Verordnungscharakter. Diese Erweiterung der Klagemöglichkeiten von Einzelnen ist nicht zuletzt auch als notwendige Antwort auf die vom Gerichtshof Erster Instanz im Zusammenhang mit den vom Rat zum Zweck finanzieller Sanktionen erstellten ,Terroristenlisten “37 festgestellte Rechtsschutzlücke im Bereich der ,dritten Säule` zu werten. ${ }^{38}$

Die Möglichkeiten richterlichen Schutzes der Rechte des Einzelnen werden schließlich auch durch die rechtsverbindliche Eingliederung der Charta der Grundrechte gestärkt. Dies gilt insbesondere für einige RFSR-Materien von bislang geringerer Schutzdichte hinsichtlich der Tätigkeit von EU-Organen und -Strukturen. So stellt beispielsweise die recht umfassende Formulierung des Rechtes auf den Schutz personenbezogener Daten (Artikel 8 der Charta) im Hinblick auf die Proliferation von EU-Datenaustausch und -analysesystemen (SIS II, Europol, Eurodac, VIS usw.) und die sich rasch entwickelnde Zusammenarbeit mit Drittstaaten im Bereich des Datenaustauschs (ein Beispiel ist das Europol-USA Abkommen vom Dezember 2002, das den Austausch von personenbezogenen Daten vorsieht) fraglos einen Fortschritt im Sinne des Rechtsschutzes auf EU-Ebene dar. Von beträchtlicher Bedeutung für den RFSR sind auch die justiziellen Rechte in Kapitel VI der Charta. Die Charta geht unter anderem durch die Aufnahme eines Rechtes auf Prozesskostenhilfe (Artikel 47), des Grundsatzes der Verhältnismäßigkeit im Zusammenhang mit Straftaten und Strafen (Artikel 49) und eines erweitert formulierten Doppelbestrafungsverbots (Artikel 50) deutlich über eine Minimalausstattung klassischer Verteidigungsrechte hinaus. ${ }^{39}$ Auch in dieser Hinsicht könnte sich - insbesondere angesichts der erweiterten Handlungsmöglichkeiten der Union im strafrechtlichen Bereich - die Kontrollfunktion des Gerichtshofs ebenfalls als sehr wichtig erweisen.

37 Für diese wird durch Artikel 75 AEUV erstmals eine klare Rechtsgrundlage geschaffen - auch dies ein Beitrag zur Rechtssicherheit im RFSR.

38 Europäischer Gerichtshof: Rs. C-354/04 P (Gestoras pro Amnestia u.a/Rat), Slg. 2007, I-1579; und Rs. C-355/ $04 \mathrm{P}$ (Segi u.a/Rat), Slg. 2007, I-1657, insbesondere Rdnr. 51 und 53. Zur Bedeutung dieser Entscheidungen für den richterlichen Schutz im Bereich des Titels VI EUV siehe Ulrich Haltern: Rechtsschutz in der dritten Säule der EU, in: JuristenZeitung 15-16/2007, S. 772-778.

39 Zur Bedeutung der justiziellen Rechte der Charta siehe Joachim Vogel: The European Integrated Criminal Justice System and Its Constitutional Framework, in: Maastricht Journal of European and Comparative Law 2/ 2005, S. 125-147. 


\section{Verstärkte Differenzierung}

Bereits im Vertrag von Amsterdam wurden die Reformen in den Bereichen Inneres und Justiz unter dem Leitziel des RFSR durch die ,Opt-outs' und ,Opt-in'-Regelungen zugunsten des Vereinigten Königreichs, Irlands und Dänemarks in den vergemeinschafteten Bereichen Asyl, Einwanderung, Grenzkontrollen und zivilrechtliche Zusammenarbeit, erkauft ${ }^{*}{ }^{40}$ Der Vertrag von Lissabon behält nicht nur diese bestehenden Ausnahmeregelungen für die drei genannten Mitgliedstaaten vollauf bei, sondern dehnt diese - mit einer Reihe von prozeduralen Veränderungen und Variationen ${ }^{41}$ - auf die gegenwärtige ,dritte Säule‘ aus. Die somit auf die polizeiliche und justizielle Zusammenarbeit in Strafsachen erweiterten ,Optouts ' sind Teil der Konzessionen, die in den Verhandlungen zur Sicherstellung der Zustimmung dieser drei Mitgliedstaaten zur faktischen, Vergemeinschaftung ' der Felder des Titels VI EUV (mit Mehrheitsentscheidung und Mitbestimmung des Parlaments) gemacht wurden.

Die gewährten ,Opt-in'-Möglichkeiten bieten den ,Opt-outs' ein großes Maß von Flexibilität hinsichtlich ihrer Beteiligung am RFSR: Sie können sich nicht nur während des Gesetzgebungsverfahrens jederzeit für eine Beteiligung (einen ,Opt-in') erklären, sondern auch - wenn sich ihre Auffassung später ändert - nach der Annahme des betreffenden Aktes durch die anderen Mitgliedstaaten auf der Grundlage der dafür vorgesehenen Verfahrensweisen. Von dieser Möglichkeit haben Irland und das Vereinigte Königreich bislang einen zwar selektiven, aber insgesamt doch reichlichen, Gebrauch gemach $t^{42}$ und es ist davon auszugehen, dass beide Länder ähnlich auch mit dem erweiterten ,Opt-out ‘ umgehen würden. Dänemark muss sich faktisch aufgrund seiner Schengen-Mitgliedschaft ohnehin an allen auf den Schengen-Besitzstand aufbauenden Rechtsakten beteiligen. ${ }^{43}$ Aber auch wenn die erweiterten ,Opt-outs' in der Praxis weniger umfassend ausfallen sollten, bleibt dennoch die Tatsache, dass mit dem neuen Vertrag drei Mitgliedstaaten nunmehr einen im Prinzip den gesamten RFSR umfassenden ,Opt-out' erhalten haben. Dies gestaltet nicht nur die Entscheidungsverfahren, die institutionelle Entwicklung und die Verhandlungen mit Drittstaaten schwieriger - oft stellen sich schwierige Abgrenzungsfragen hinsichtlich der Beteiligung oder Nichtbeteiligung der ,Opt-outs` ${ }^{*}$, sondern geht auch zu Lasten der Glaubwürdigkeit des RFSR als grundlegendes Vertrags- und Integrationsziel sowie auch der einheitlichen internationalen Handlungsfähigkeit der Union in diesem Bereich.

Damit ist das neue Differenzierungspotenzial aber noch nicht erschöpft. Wie bereits erwähnt sieht der neue Vertrag einen stark erleichterten Übergang zur ,,verstärkten Zusammenarbeit" in den folgenden Fällen vor: falls nach Aktivieren der Notbremse bei Richtlinien zur Festlegung von Mindestvorschriften im Bereich des Strafverfahrensrechts (Artikel 82(3) AEUV) oder bei Richtlinien zu Mindestvorschriften zur Festlegung von Straftaten und Strafen in Bereichen besonders schwerer Kriminalität (Artikel 83(3) AEUV) im Europäischen Rat keine Einigung erzielt werden kann und bei erfolglosem Bemühen um Einstimmigkeit

40 Siehe hierzu Pierre Berthelet: Le droit institutionnel de la sécurité intérieure européenne, Brüssel 2003, S. 215271; und Jörg Monar: Justice and Home Affairs in the Treaty of Amsterdam: Reform at the Price of Fragmentation, in: European Law Review 4/1998, S. 320-335.

41 Der dänische, Opt-out ' wird sich beispielsweise nicht auf die Festlegung der Bedingungen und Grenzen innerhalb derer Polizeibehörden der Mitgliedstaaten im Hoheitsgebiet eines anderen Mitgliedstaats in Verbindung und in Absprache mit dessen Behörden tätig werden dürfen (Artikel 89 AEUV) erstrecken. Anscheinend wurde von der dänischen Regierung die Beibehaltung der Einstimmigkeit im Rat und die bloße Anhörung des Parlaments für hinreichende Gewährleistungselemente der Zwischenstaatlichkeit erachtet, um auf eine Anwendung des ,Opt-outs' auch auf diesen Bereich verzichten zu können.

42 Siehe Steve Peers: Vetoes, Opt-outs and EU Immigration and Asylum law, Statewatch briefing, 2004.

43 Diese sind dann für Dänemark allerdings nur nach internationalem Recht und nicht nach EG-Recht verbindlich. 
hinsichtlich der Einsetzung einer Europäischen Staatsanwaltschaft (Artikel 86(1) AEUV) oder der Maßnahmen zur operativen Zusammenarbeit zwischen den Polizeibehörden der Mitgliedstaaten (Artikel 87(3) AEUV). In allen diesen Fällen kann eine Gruppe von mindestens neun Mitgliedstaaten dem Europäischen Parlament, dem Rat und der Kommission einfach mitteilen, dass sie die betreffenden Rechtsakte auf dem Wege einer ,,verstärkten Zusammenarbeit" annehmen möchte, woraufhin eine entsprechende Ermächtigung als gegeben gilt.

Diese Möglichkeiten der ,,verstärkten Zusammenarbeit“ sind im Grunde nur eine Variante - obwohl eine potenziell konstruktivere - der den RFSR mehr und mehr dominierenden Logik des Verfolgens separater Interessen, die auch den ,Opt-outs ‘ zugrunde liegt. Während sich im Falle der ,Opt-outs“ einige Mitgliedstaaten das Recht vorbehalten haben, nur an den RFSR weiter entwickelnden Maßnahmen teilzunehmen, die ihren Interessen entsprechen, bietet die „,verstärkte Zusammenarbeit“ Gruppen von Mitgliedstaaten die Möglichkeit, gewisse Maßnahmen ohne die Unterstützung und Beteiligung der anderen Mitgliedstaaten umzusetzen und gegebenenfalls auch vorzuschlagen. ${ }^{44}$ Obwohl der RFSR ein gemeinsames, durch den neuen Vertrag sogar noch aufgewertetes Vertragsziel darstellt, wird dieser damit zumindest in Teilen potenziell zu einem Projekt für nur einige Mitgliedstaaten, das folglich nicht allen Bürgern der Union überall in der Union dasselbe Ausmaß von „Freiheit, Sicherheit und Recht" bietet. Geht man beispielsweise davon aus, dass EU-Maßnahmen zur Verbesserung der operativen Zusammenarbeit zwischen den Polizeibehörden der Mitgliedstaaten gemäß Artikels 87(3) AEUV zur Erreichung des Zieles ,eines hohen Maßes an Sicherheit“ (Artikel 67(3) AEUV) beitragen, so würde eine Nutzung der ,verstärkten Zusammenarbeit" für diese Maßnahmen dieses ,mehr" an Sicherheit nur (oder zumindest primär) für die Bürger der beteiligten Mitgliedstaaten erbringen und in diesem Sinne der RFSR nicht gleichermaßen allen Bürgern ,geboten“ werden.

\section{Schlussfolgerungen}

Der Vertrag von Lissabon enthält eine ganze Reihe von Reformen, die zu einer größeren Handlungsfähigkeit der Union hinsichtlich Entscheidungsverfahren, Kompetenzen und Umsetzungsfähigkeit beitragen können. Mindestens ebenso bedeutsam - und zur Sicherung der Legitimation eines mehr und mehr expandierenden RFSR notwendiger - ist die Ausweitung der parlamentarischen und richterlichen Kontrollmöglichkeiten. Sollte der Vertrag definitiv scheitern, so würde dies zweifellos die Entwicklungsmöglichkeiten des RFSR und die Effizienz der Innen- und Justizpolitik der Union in erheblichem Maße reduzieren und - vielleicht für den Bürger noch beunruhigender - das Fortbestehen erheblicher Defizite der parlamentarischen und richterlichen Kontrolle bedeuten.

Dennoch muss man auch klar sehen, dass der Vertrag von Lissabon nicht nur längst nicht alle bestehenden Entwicklungsprobleme des RFSR lösen, sondern einige sogar noch verstärken würde: Die Fortschritte hinsichtlich Entscheidungsverfahren, Kompetenzen und Umsetzungsfähigkeit sind in einem Ausmaß mit Ausnahmen und Beschränkungen durchsetzt, dass die möglichen Effizienzgewinne teilweise wieder reduziert und dem - im Vertrag offenkundig ohnehin abhanden gekommenen - Grundsatz der Transparenz Hohn spricht. Zudem ist

$44 \mathrm{Zu}$ berücksichtigen ist hier, dass der Vertrag von Lissabon - wie bereist erwähnt - im Bereich der polizeilichen und justiziellen Zusammenarbeit in Strafsachen die Möglichkeit von Gesetzgebungsinitiativen seitens (mindestens) eines Viertels der Mitgliedstaaten vorsieht. Dies eröffnet die Möglichkeit, dass eine 9+-Gruppe eine Gesetzgebungsinitiative einbringt, die von Anfang an so formuliert ist, dass sie für andere Mitgliedstaaten völlig unannehmbar ist und folglich einen direkten Weg zur Autorisierung einer entsprechenden ,,verstärkten Zusammenarbeit" ebnet. 
der Preis, der von den ,Vätern der Verträge‘ für die erzielten Fortschritte in Gestalt von monströsen Verfahrensvarianten und stark erhöhtem Differenzierungspotenzial gezahlt wurde noch höher als im Vertragswerk von Amsterdam, sodass man sich die Frage stellen muss, wie sehr der RFSR noch als wirkliches, gemeinsames‘ Projekt der Union gelten kann.

Ob der Vertrag nun aber in Kraft tritt oder nicht, wird sich die Union in jedem Fall mit den Gegenständen der Vertragsreformen in den nächsten Jahren befassen müssen: Zumindest einige der neuen Zielsetzungen im asylpolitischen, strafrechtlichen und polizeilichen Bereich sind - bei genügend politischem Willen - auch auf der Grundlage der gegenwärtigen Vertragsbestimmungen erreichbar, und hinsichtlich der Entscheidungsverfahren und der parlamentarischen und richterlichen Kontrolle könnte notfalls der Vorschlag der Kommission von 2005 zur Nutzung der ,Passerelle “ wieder aufgegriffen werden. Von beträchtlicher Bedeutung wird im nächsten Jahr die Einigung auf ein gehaltvolles und dynamisches Nachfolgeprogramm 2010-2014 für das gegenwärtige Haager Programm sein, für die die Arbeiten der hochrangigen „Zukunftsgruppe“ und ihr Bericht vom Juli $2008^{45}$ bereits wichtige Akzente gesetzt haben. Der Vertrag von Lissabon würde das Entwicklungspotenzial des RFSR gewiss stärken, aber sein eventuelles Scheitern muss durchaus nicht - und darf im Interesse der Unionsbürger auch nicht - zu einer Stagnation des RFSR führen.

45 Bericht der Informellen Hochrangigen Beratenden Gruppe zur Zukunft der Europäschen Innenpolitik (,,Zukunftsgruppe"): Freiheit, Sicherheit, Privatheit - Europäische Innenpolitik in einer offenen Welt, Berlin (Bundesministerium des Innern), Juli 2008. Zu den Arbeiten der Zukunftsgruppe siehe Matthias Oel/Juliane RappLücke: Politische Entscheidungsvorbreitung in der EU-27 plus am Beispiel der europäischen Innenpolitik, in: integration 3/2008, S. 281-289. 